\title{
L'emodialisi domiciliare: come per la ribollita, da un obbligo per necessità a una scelta ristretta per intenditori
}

\author{
Giancarlo Ruggieri
}

\section{Dipartimento di Nefrologia e Urologia, Centro di Riferimento di Nefrologia e Dialisi, Ospedale di San Giacomo in Augusta, Roma}

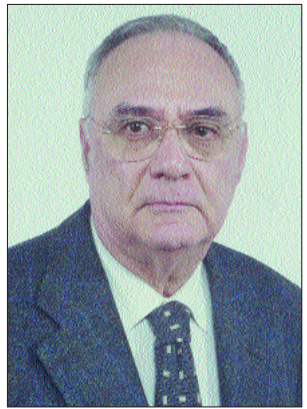

A una prima valutazione sembrerebbe non facile interpretare il fenomeno della progressiva riduzione, fino praticamente a una scomparsa, dell'emodialisi domiciliare, fenomeno a carattere per così dire universale. In apparenza, tutto oggi sembrerebbe indicare l'esistenza di condizioni quanto mai favorevoli all'adozione di questa terapia: la visione della Sanità nel pubblico e nei media, che esprime una forte sollecitazione verso l'automedicazione e il controllo responsa- bile, altamente personalizzato e cosciente, delle proprie condizioni morbose, i progressi tecnologici, che hanno dato all'emodialisi apparecchiature altamente sofisticate e capaci di controllare e guidare in modo automatico e sicuro profili anche molto complessi di terapia dialitica, sempre più personalizzabili e con profili strategici sempre più differenziati, la letteratura scientifica, con gli eccellenti risultati ottenibili dall'emodialisi quotidiana e la possibilità tecnica di moderarne la spesa, e infine l'economicità delle terapie domiciliari in generale, realizzata anzitutto dal vantaggio sociale e dalla capacità di tutela del lavoro. Se però si esamina l'utilizzo delle diverse terapie dialitiche nel corso del tempo, la letteratura ri- guardante l'emodialisi domiciliare all'epoca della sua maggiore diffusione, e quanto è accaduto in seguito, $\mathrm{i}$ motivi dell'attuale situazione dell'emodialisi domiciliare appaiono assai più chiari di quanto possa sembrare a primo sguardo.

La Tabella qui inserita indica l'andamento di alcuni parametri fondamentali dell'attività dialitica negli anni compresi fra il 1973 e il 1990 (elaborazione da dati ANED).

Dai dati della Tabella è possibile vedere come nel periodo 1973-1980 (anno in cui la EDD raggiungeva l'acme di utilizzo per milione d'abitanti) i posti dialisi erano cresciuti a un ritmo medio annuo inferiore a quello delle persone da dializzare $(8.78 \%$ contro il $10.34 \%)$ mentre la

TABELLA I

\begin{tabular}{rrrrrrrrrrrrrrrrrrr}
\hline $\mathbf{1 9 7 3}$ & $\mathbf{1 9 7 4}$ & $\mathbf{1 9 7 5}$ & $\mathbf{1 9 7 6}$ & $\mathbf{1 9 7 7}$ & $\mathbf{1 9 7 8}$ & $\mathbf{1 9 7 9}$ & $\mathbf{1 9 8 0}$ & $\mathbf{1 9 8 1}$ & $\mathbf{1 9 8 2}$ & $\mathbf{1 9 8 3}$ & $\mathbf{1 9 8 4}$ & $\mathbf{1 9 8 5}$ & $\mathbf{1 9 8 6}$ & $\mathbf{1 9 8 7}$ & $\mathbf{1 9 8 8}$ & $\mathbf{1 9 8 9}$ & $\mathbf{1 9 9 0}$ \\
$\mathbf{1}$ & 2.5 & 3.6 & 5.4 & 8.1 & 10.9 & 13.5 & 16.3 & 17.3 & 17.1 & 16.9 & 16.3 & 15.6 & 14.3 & 13.2 & 12.6 & 11.5 & 10.8 & 9.9 \\
$\mathbf{2}$ & 62.3 & 79.3 & 99.2 & 124.3 & 147.5 & 173.4 & 200.3 & 226 & 256.6 & 283.2 & 307.2 & 350.8 & 355.1 & 377.9 & 400.7 & 424.6 & 447.1 & 470.4 \\
$\mathbf{3}$ & 28 & 34.4 & 39.1 & 44.3 & 56.9 & 57.3 & 64.3 & 72.8 & 80.3 & 86.6 & 94.1 & 100.8 & 100.6 & 116 & 124.5 & 130.7 & 137.6 & 144.9 \\
$\mathbf{4}$ & 2.2 & 2.3 & 2.5 & 2.8 & 2.9 & 3 & 3.11 & 3.1 & 3.2 & 3.3 & 3.3 & 3.3 & 3.3 & 3.2 & 3.2 & 3.2 & 3.2 & 3.2 \\
$\mathbf{5}$ & - & - & 0.07 & 0.11 & 0.12 & 0.26 & 1.99 & 7.26 & 12.8 & 17.3 & 19.2 & 22.1 & 25 & 26.9 & 29.2 & 31.3 & 35 & 38.3
\end{tabular}


dialisi peritoneale iniziava il suo ingresso clinico tangibile solo nel 1979: nel 1980 la dialisi domiciliare trattava il $10.8 \%$ dei pazienti. Nel successivo decennio 1980-1990 i posti dialisi crescono più rapidamente della popolazione in dialisi (il $4.8 \%$ verso il $4.1 \%$ ), l'utilizzo dei posti dialisi si stabilizza sul più soddisfacente indice di 3.3-3.2: le tecniche domiciliari (EDD + DP) trattano nel 1990 il $10.77 \%$ delle persone in dialisi $(48.2$ verso 474.4 per milione d'abitanti): in quello stesso decennio si verifica il crollo della EDD, che scende rapidamente, raggiungendo nel 1990 il $41.4 \%$ del valore 100 ottenuto nel 1980 (17.3 trattati per milione d'abitanti), corrispondente a una percentuale del $2.08 \%$ su tutti i pazienti trattati. Dagli ultimi dati disponibili nel sito SIN del Registro Italiano di Dialisi e Trapianto per il 1998, l'emodialisi domiciliare non è neanche censita come tale, e, salvo personali errori di lettura, sembrerebbe possibile intravederne la dimensione attuale dalla differenza esistente fra la percentuale del $12.5 \%$ della dialisi domiciliare su tutta la dialisi (Tabella Dialysis Facilities) e la grandezza percentuale dell' $11.5 \%$ della dialisi peritoneale CAPD + APD, da ritenersi attribuibile a peritoneale domiciliare: la EDD sarebbe quindi pari al $12.5-11.5 \%=$ $1 \%$ quindi, riguardante 313 persone pari a $6.3 / 10^{6}$ abitanti delle Regioni censite.

Perché questo abbandono di una terapia il cui utilizzo era maggiore allorquando la sua effettuazione era certamente più difficile e a rischio di quanto può essere oggi? La prima risposta sta nell'esame dei motivi che spingevano i nefrologi attivi negli anni precedenti il 1980 alla scelta dell'emodialisi domiciliare. In occasione del II Corso del San Carlo (1970), il Dott. Romagnoni della Nefrologia del San Carlo stesso dà le fondamentali ragioni dell'epoca, «scopo della dialisi è il trattamento del maggior numero di pazienti... in modo da consentire il massimo recupero psicofisico e sociale, contenendo al massimo l'onere finanziario che la società deve sobbarcarsi... La realizzazione può venire raggiunta con un numero sem- pre maggiore di Servizi di dialisi... (ciò) non si rivela attuabile perché ... difficoltà di reperimento del personale... elevati costi... La soluzione ospedaliera non riesce a ovviare al grave problema delle infezioni batteriche e dell'epatite virale... Il ruolo del Centro dialisi è quello di addestratore... (La dialisi domiciliare) può essere praticata nelle ore notturne, permettendo il sonno... lasciando al paziente tutte le ore diurne della settimana...». Queste motivazioni esprimono concetti che suonano modernissimi anche oggi per quanto attiene il contenimento della spesa e il vantaggio del paziente per il lavoro e la sua libertà (dialisi notturna) e difatti vengono continuamente usate per la APD. Ma le parole di Romagnoni contengono anche i gravi problemi dell'epoca legati alla difficoltà di reperire le risorse economiche, tecniche e umane per sopperire al crescente numero di uremici che ricorrevano alla dialisi, ormai realtà clinica: come visto dalla Tabella, il numero dei dializzati saliva più rapidamente del numero dei posti dialisi, la cui utilizzazione era assai limitata dalle tecniche disponibili e dalla conseguente lunga durata della seduta (8-12 ore). A questo si associava l'elevato rischio infettivo della dialisi ospedaliera, che per quanto riguardava l'epatite virale coinvolgeva spesso anche il personale addetto. Da tutto questo, il ricorso quando possibile al trattamento domiciliare diveniva una via obbligata e quindi poteva/doveva essere affermato «... Il ruolo del Centro dialisi è quello di addestratore...».

Queste necessità e questa visione nell'erogare la dialisi sono ben presenti nella letteratura dei primi 10-15 anni di dialisi clinica (a partire dai primi anni '60), in alcune aree anche per l'estensione del territorio (USA, Canada e Australia): Yukihico Nosè ha recentemente ricordato la necessità assoluta di praticare in USA l'emodialisi domiciliare già nel 1963, ripresa poi malgrado i rischi nel 1965, per il numero di persone che morivano per mancanza di posti ospedalieri: McBride (Aust NZJ Med, Oct, 5, $401,1975)$ in un calcolo di programma dell'assistenza in dialisi preconiz- zava un raddoppio della disponibilità per l'emodialisi domiciliare contro un aumento del $50 \%$ per i posti ospedalieri. A fianco di questa impostazione su esigenze di base medica ed economica, erano però presenti in letteratura osservazioni a carattere psicologico e sociale, le quali rilevavano pazienti e famiglie che avevano ben integrato la dialisi a domicilio nella loro vita, ma anche situazioni di stress e sofferenza, una possibile influenza negativa sull'adolescenza di ragazzi con un genitore in dialisi domiciliare, il carico che ricadeva sul mondo femminile (personalmente riscontrato anche in dialisi peritoneale) e soprattutto rilevavano anche «una carenza di un sistema di servizio di supporto continuativo» (Kalodner, J Am Geriatr Soc, Jan 24, 1, 32, 1976), e inoltre anche la mancanza di cura dei medici che oggi diremmo di famiglia e del servizio sanitario in genere: queste osservazioni si facevano maggiormente frequenti a partire dalla seconda metà degli anni ' 70 , mentre veniva segnalata l'opportunità di istituire ambulatori decentrati per trattare le persone che mal tolleravano l'emodialisi domiciliare, cui era stata anche imputato di causare una sorta di segregazione sociale. In questa situazione di non totale soddisfazione, l'ipotesi di irrealizzabilità di una sufficiente rete di posti dialisi nel territorio si manifestava errata, e i posti dialisi crescevano rapidamente mentre le tecniche consentivano un uso assai più intensivo del posto dialisi: in Italia questo si verificava nel Centro Nord per il sorgere, oltre che di nuovi servizi di nefrologia ospedalieri, di una sempre più elevata disponibilità di Centri di dialisi ad assistenza limitata, e nel Centro Sud per la proliferazione incontrollata di Centri dialisi profit a rimborso statale. Questa mutata condizione, affiancata dallo sviluppo della dialisi peritoneale (vedi avanti), peraltro limitata quasi del tutto al Nord Italia, spegneva il più importante motivo dei nefrologi a ricorrere all'emodialisi domiciliare, e lo stesso eventuale interesse personale del malato, se non assai fortemente motivato, perdeva ogni vivacità di fronte alla disponibilità di una dialisi 
senza carichi personali effettuata a distanza ragionevole dalla sua residenza o luogo di lavoro. La dialisi peritoneale ha giocato un ruolo variabile nell'abbattimento dell'emodialisi domiciliare, a seconda delle aree. Dati EDTA relativi al periodo 1981-1988 evidenziano come l'Italia in quegli anni si sia comportata in modo parallelo a quello medio dell'Europa: i fenomeni di crescita della dialisi peritoneale e di discesa dell'emodialisi domiciliare, pure se simultanei, appaiono non correlati in quanto l'HDD continua a scendere fino al 1988 sebbene la PD avesse in pratica arrestato la sua crescita dal 1985: nei fatti la dialisi domiciliare nel suo insieme cade o non si sviluppa mentre cresce continuamente l'emodialisi ambulatoriale; per converso i dati del Regno Unito e dei Paesi Nordici dimostrano inconfutabilmente la sostituzione dell'una tecnica con l'altra mentre la Francia poco modifica l'HDD e poco sviluppa la PD. Non può peraltro essere negletto il fatto (Kendix, 1995 Schlesinger, 1989) che l'offerta di posti dialisi, ove eccessiva, comprime al di sotto del ragionevole le terapie domiciliari, anche per l'evidente interesse dei Centri profit e oggi forse non solo di questi a fornire le prestazioni nel Centro dialisi, perché più remunerative malgrado i costi più elevati.

Le possibilità di ripresa dell'emodialisi domiciliare, oltre che da un crescente diffuso apprezzamento dei vantaggi evidenziatisi di recente nei riguardi dello stato nutrizionale, della condizione ipertensiva e cardiaca con l'emodialisi lunga notturna, dipendono però e anzitutto, esattamente come per la dialisi peritoneale, da una presa di coscienza dei governi nazionale e regionali del crescente gravame economico derivante dal crescente numero dei dializzati, se trattati quasi esclusivamente con le forme più costose di dialisi e che generano anche maggior costo sociale indotto, con la conseguente assunzione di direttive sollecitanti un più razionale utilizzo delle risorse economiche. 